\title{
AN ORIENTATION REVERSING HOMEOMORPHISM OF THE PLANE WITH INVARIANT PSEUDO-ARC
}

\author{
DAVID P. BELLAMY AND WAYNE LEWIS
}

(Communicated by James E. West)

\begin{abstract}
Using the universal cover of the Möbius band, we construct an example as described in the title which answers a question of $\mathrm{K}$. Kuperberg.
\end{abstract}

Krystyna Kuperberg has recently asked whether there exists an orientation reversing homeomorphism of the plane with an invariant pseudo-arc. We provide a positive answer to this question. Her question was apparently motivated by her recent work [4] on fixed points and orientation reversing homeomorphisms.

Most embeddings of the pseudo-arc in the plane appear to admit very few homeomorphism which extend to homeomorphisms of the plane. For many embeddings, e.g., the "standard" embedding with two accessible composants [7], one can show that every extendable homeomorphism must preserve orientation of the plane. Thus our example depends on choosing the correct embedding of the pseudo-arc.

Since there has been significant recent interest in the dynamics of homeomorphisms of the pseudo-arc and in which homeomorphisms of the pseudo-arc extend to homeomorphisms of the plane, it is worth noting that the example we construct has fairly elementary dynamics. There are two fixed points of the pseudo-arc under our homeomorphism. Every other point of the psuedo-arc is repelled from one of these fixed points and attracted to the other. One can construct examples with more interesting dynamics.

We conclude with some observations about prime ends, accessible points, and extendable homeomorphisms.

\section{THE CONSTRUCTION}

Our main result is the following

Theorem. There exists an orientation reversing homeomorphism of the plane with an invariant pseudo-arc.

Our proof is constructive. The construction can be outlined in the following few steps and observations.

Received by the editors June 19, 1990.

1991 Mathematics Subject Classification. Primary 54H20, 54F50; Secondary 57M10.

Key words and phrases. Covering map, invariant continuum, Möbius band, pseudo-arc, pseudocircle.

The second author was supported by Texas Advanced Research Program grant 88-1296. 
1. There exists an essential degree one embedding of the pseudo-circle in the Möbius band.

2. The universal cover of the Möbius band is homeomorphic to $[0,1] \times \mathbb{R}$.

3. A deck transformation produces an orientation reversing homeomorphism of the universal cover, which is invariant on the lift of the pseudo-circle.

4. The two-point compactification of the universal cover is a disc, with the lift of the pseudo-circle compactifying to a spanning pseudo-arc.

5. The deck transformation in step 3 extends to an orientation reversing homeomorphism of the disc with an invariant spanning pseudo-arc.

6. This homeomorphism of a disc extends to an orientation reversing homeomorphism of the plane with an invariant pseudo-arc.

All steps except 1 and 4 are straightforward, thus we confine our attention to these two steps. For basic facts about the pseudo-arc, pseudo-circle, and crooked chains, the reader is referred to $[1,2,3,6,8]$.

Step 1. The pseudo-circle is characterized [3] as a circularly chainable, nonchainable, hereditarily indecomposable continuum which can be embedded in the plane. To embed it in the Möbius band, it is sufficient to form an appropriate sequence of circular chains. It is convenient to think of the Möbius band as $[0,1] \times[0,1]$ with $\{0\} \times[0,1]$ identified with $\{1\} \times[0,1]$ (with order reversed on the second factor). For the argument in step 4 we will think of its universal cover as $\mathbb{R} \times[0,1]$ with the covering map preserving the first coordinate $(\bmod \mathbb{Z})$.

In $[0,1] \times[0,1]$ consider the three straight line segments joining (a) $\left(0, \frac{1}{4}\right)$ to $\left(1, \frac{1}{3}\right),(\mathrm{b})\left(1, \frac{1}{3}\right)$ to $\left(0, \frac{1}{2}\right)$, and $(\mathrm{c})\left(0, \frac{1}{2}\right)$ to $\left(1, \frac{3}{4}\right)$. Under identification these form a simple closed curve going once essentially around the Möbius band. This simple closed curve is to be the centerline for a circular chain $D_{1}$ of small mesh. The links of this circular chain are to be chosen to be discs, with the intersection of any two adjacent links a disc, such that their union forms a thin Möbius band inside the original.

The circular chain $D_{1}$ consists of two parts. One covers the union of the line segments in (c) and (a) and goes around the original Möbius band twice. The other covers the line segment in (b) and goes around the Möbius band once in the opposite direction. The circular chain $D_{2}$ refines $D_{1}$ and consists of the union of two chains, one of which is crooked in the part of $D_{1}$ covering the line segments in (c) and (a) and the other of which is crooked in the part of $D_{1}$ covering the line segment in (b). $D_{2}$ goes essentially once around $D_{1}$ and its links are chosen to be discs, with the intersection of any adjacent links a disc such that their union is a thin Möbius band.

In general the circular chain $D_{2 n}$ consists of two pieces, a long crooked piece and a short crooked piece. To form the chain $D_{2 n+1}$ one first forms three arcs in the pattern of (a), (b), and (c) in $D_{2 n}$, starting with (a) first running through the long crooked piece then the short crooked piece, with the same orientation as in the previous steps. These are then used as the centerline for the circular chain $D_{2 n+1}$. The circular chain $D_{2 n+2}$ is the union of two chains, one crooked in the part of $D_{2 n+1}$ covering the (c) and (a) arcs, the other crooked in the part of $D_{2 n+1}$ covering the (b) arc.

Continuing in this manner, with proper control on mesh, the intersection of the regions covered by the sequence of circular chains forms a pseudo-circle. 
Step 4. Each of the circular chains $D_{i}$ in Step 1 lifts to an infinite chain $C_{i}$ in the universal cover of the Möbius band. With appropriate choices of neighborhoods of the two compactification points and finite segments of the $C_{i}$ 's one obtains chains of arbitrarily small mesh covering the compactification of the lift of the pseudo-circle. Thus this compactification is a chainable continuum. To show that is is a pseudo-arc we need only show that it is hereditarily indecomposable [2]. For this it suffices to show that for any finite segment $C$ of any $C_{i}$ there exists $k>i$ such that each subchain of $C_{k}$ contained in $C$ is crooked in $C$. We show this by induction.

Assume that the finite segment $C$ is contained in $[m, m+j] \times[0,1]$, where $m$ and $j$ are chosen such that this is the minimal interval covering $C$. We induct on $j$. Our inductive hypothesis is that a copy of the lift of each of the (a), (b), and (c) segments of $D_{2 j-1}$ runs the length of $[m, m+j] \times[0,1]$, with leftmost end link at $\{m\} \times[0,1]$ and rightmost end link at $\{m+1\} \times[0,1]$. By construction of $D_{1}$, this is clearly true for $j=1$.

Suppose it is true for $j=j_{0}$. One copy of the lift of the (c) segment of $D_{2 j_{0}-1}$ spans $\left[m, m+j_{0}\right] \times[0,1]$, with end links at $\{m\} \times[0,1]$ and $\{m+1\} \times[0,1]$. The next copies of the lifts of the (a) and (b) segments of $D_{2 j_{0}-1}$ each span $\left[m+1, m+1+j_{0}\right] \times[0,1]$, each with end links in $\{m+1\} \times[0,1]$ and $\{m+2\} \times[0,1]$. The lift of the (c) segment of $D_{2 j_{0}-1}$ intersects the next lift of the (a) segment of $D_{2 j_{0}-1}$ in their end links at $\{m+1\} \times[0,1]$, while the lifts of the (a) and (b) segments of $D_{2 j_{0}-1}$ intersect in their end links at $\{m+2\} \times[0,1]$. Thus the chain formed by one copy of the lift of the (c) segment of $D_{2 j_{0}-1}$ and the next copies of the lifts of the (a) and (b) segments of $D_{2 j_{0}-1}$ spans [ $\left.m, m+1+j_{0}\right] \times[0,1]$ with end links at $\{m\} \times[0,1]$ and $\{m+1\} \times[0,1]$. Copies of the lifts of each of the (a), (b), and (c) segments of $D_{2\left(j_{0}+1\right)-1}$ span the length of this chain with ends in its links, thus satisfying our inductive hypothesis for $j=j_{0}+1$.

Any subchain of $C_{2(j+1)}$ that is contained in $[m, m+j] \times[0,1]$, with $m$ and $j$ chosen to be minimal with this property, is contained in one of the following portions of $C_{2(j+1)-1}$ :

(i) one lift of the (a) segment of $D_{2(j+1)-1}$ and the preceding lift of the (c) segment,

(ii) parallel lifts of the (a) and (b) segments of $D_{2(j+1)-1}$, or

(iii) one lift of the (c) segment of $D_{2(j+1)-1}$ and the succeeding lift of the (a) segment.

In each of cases (i) and (iii) the subchain of $C_{2(j+1)}$ is crooked in the corresponding part of $C_{2(j+1)-1}$ by the construction of $D_{2(j+1)}$. In case (ii) the part of the subchain of $C_{2(j+1)}$ in the lifts of each of the (a) and (b) segments of $D_{2(j+1)-1}$ is crooked in the corresponding lift. Since these segments are parallel in $C_{2 j}$ and intersect only at ends, the entire subchain of $C_{2(j+1)}$ is crooked in the corresponding part of $C_{2 j}$.

Thus, if $C$ is contained in $[m, m+j] \times[0,1]$ it is sufficient to choose $k=\max \{i+3,2(j+1)\}$. Our construction and proof are now complete.

\section{FURTHER OBSERVATIONS}

Our proof of Step 4 demonstrates that any Hausdorff two-point compactification of the universal cover of the pseudo-circle must have the compactication 
points located one at the $-\infty$ end of the cover and one at the $+\infty$ end of the cover, since the lift of any chain $D_{2 j-1}$ is an infinite chain with a bound on how far it bends back. Thus our argument will also yield that it is chainable and hereditarily indecomposable. This yields the following fact in which there has been some interest.

Fact. Any Hausdorff two-point compactification of the universal cover of the pseudo-circle is homeomorphic to the pseudo-arc.

Now we comment on prime ends, accessible points, and embeddings of the pseudo-arc. Associated with each embedding of the pseudo-arc in the plane is its space of prime ends, homeomorphic to a simple closed curve. Each homeomorphism of the pseudo-arc which extends to a homeomorphism of the plane induces a homeomorphism on the associated space of prime ends. The homeomorphism on the space of prime ends preserves orientation if and only if the homeomorphism of the plane preserves orientation. Each accessible point of the pseudo-arc is associated with a prime end. For two distinct accessible points, there are in the space of prime ends two arcs joining the prime ends corresponding to these accessible points. If these accessible points are in the same composant of the pseudo-arc, one of these two arcs has the property that every other accessible point corresponding to a prime end in this arc is also in the same composant. Thus the space of prime ends is in general composed of intervals corresponding to the composants of accessible points. Any homeomorphism of the pseudo-arc which extends to a homeomorphism of the plane induces a homeomorphism of the space of prime ends that preserves these intervals.

For example, in the "standard" embedding of the pseudo-arc in the plane with two accessible composants the space of prime ends is the union of two half-open intervals corresponding to these accessible composants. Any extendable homeomorphism of the pseudo-arc induces on the space of prime ends a homeomorphism which either preserves or interchanges these two half-open intervals. Thus it must preserve orientation. Similar observations can be made for several other embeddings. Michel Smith [9] and the second author [5] have independently constructed a variety of embeddings of the pseudo-arc in the plane. Extendability of homeomorphisms for various embeddings is an area fresh for further studies.

Note. After submitting this paper, the authors learned from Marcy Barge that he has an alternate construction that also yields an example with the properties described in our title.

\section{REFERENCES}

1. R. H. Bing. A homogeneous indecomposable plane continuum, Duke Math. J. 15 (1948), 729-742.

2. ___ Concerning hereditarily indecomposable continua, Pacific J. Math. 1 (1951), 43-51.

3. L. Fearnley, The pseudo-circle is not homogeneous, Bull. Amer. Math. Soc. 75 (1969), 554558.

4. K. Kuperberg, $A$ lower bound for the number of fixed points of orientation reversing homeomorphisms, preprint.

5. W. Lewis, Embeddings of the pseudo-arc in $E^{2}$, Pacific J. Math. 93 (1981), 115-120.

6. _ The peudo-arc, Contemp. Math. 117 (1991) 103-123. 
7. E. E. Moise, An indecomposable plane continuum which is homeomorphic to each of its nondegenerate subcontinua, Trans. Amer. Math. Soc. 63 (1948), 581-594.

8. J. T. Rogers, Jr., The pseudo-circle is not homogeneous, Trans. Amer. Math. Soc. 148 (1970), 417-428.

9. M. Smith, Plane indecomposable continua, prime ends, and embeddings of the pseudo-arc, Topology Proc. 3 (1978), 295-300.

Department of Mathematics, University of Delaware, Newark, Delaware 19716

Department of Mathematics, Texas Tech University, Lubbock, TeXas 79409-1042 